\title{
Metformin inhibits inflammation and bone destruction in collagen- induced arthritis in rats
}

\author{
Kai-Jian Fan ${ }^{1 \#}$, Jing Wu ${ }^{1 \#}$, Qi-Shan Wang ${ }^{1}$, Bing-Xin Xu ${ }^{1}$, Fu-Tao Zhao ${ }^{2}$, Ting-Yu Wang ${ }^{1}$ \\ ${ }^{1}$ Department of Pharmacy, Shanghai Ninth People's Hospital, School of Medicine, Shanghai Jiao Tong University, Shanghai, China; ${ }^{2}$ Department of \\ Rheumatology, Shanghai Ninth People's Hospital, School of Medicine, Shanghai Jiao Tong University, Shanghai, China \\ Contributions: (I) Conception and design: KJ Fan, J Wu, TY Wang; (II) Administrative support: FT Zhao, TY Wang; (III) Provision of study materials \\ or patients: FT Zhao, TY Wang; (IV) Collection and assembly of data: KJ Fan, J Wu, BX Xu, QS Wang; (V) Data analysis and interpretation: KJ \\ Fan, J Wu; (VI) Manuscript writing: All authors; (VII) Final approval of manuscript: All authors. \\ \#These authors contributed equally to this work. \\ Correspondence to: Dr. Ting-Yu Wang. Department of Pharmacy, Shanghai Ninth People's Hospital, Shanghai Jiao Tong University School of \\ Medicine, No. 639 Zhizaoju Road, Shanghai, China. Email: drtywang@163.com; Dr. Fu-Tao Zhao. Department of Rheumatology, Shanghai Ninth \\ People’s Hospital, Shanghai Jiao Tong University School of Medicine, No. 639 Zhizaoju Road, Shanghai, China. Email: ftzhao@moisten.org.
}

Background: Metformin (MF) is a widely used biguanide oral hypoglycemic agent, which has obvious anti-inflammatory and immunomodulatory effects. However, the mechanism of MF on rheumatoid arthritis (RA) remains uncertain. In this study, we investigated the therapeutic effects of MF on collageninduced arthritis (CIA).

Methods: CIA was induced in rats by intradermal injection of a mixture of bovine type II collagen and incomplete Freund's adjuvant (IFA) on day 0 and day 7 through the base of the tail. Intraperitoneal injection of MF (100 mg/kg) was given every 3 days, from day 14 for 3 weeks. The effects of MF on arthritisinduced systemic inflammation and synovitis were studied by pathological analysis of the knee joint and serological examination of peripheral blood in CIA rats. The bone protection effect of MF was studied by microscopic computed tomography (micro-CT) and histological analysis of the knee joint. The effects of MF on chondrocytes in CIA rats were studied by detecting the relevant pro-apoptotic mediators in the chondrocytes.

Results: After administration of MF in CIA rats, systemic inflammation and synovitis caused by arthritis were significantly suppressed. Histomorphometry and micro-CT analysis of the knee joint revealed that MF can protect bone by inhibiting the changes of trabecular bone in CIA rats. Histological analysis of the knee joint found that MF can inhibit osteoclast formation and degradation of the cartilage layer matrix. Detection of the relevant pro-apoptotic mediators in chondrocytes revealed that MF can significantly inhibit the apoptosis of chondrocytes in CIA rats.

Conclusions: Our study showed that MF can inhibit systemic inflammation and synovitis and plays a role in bone protection by inhibiting cartilage layer matrix degradation, osteoclast formation, and chondrocyte apoptosis.

Keywords: Metformin (MF); inflammation; bone destruction; rheumatoid arthritis (RA)

Submitted Apr 01, 2020. Accepted for publication Sep 25, 2020.

doi: 10.21037/atm-20-3042

View this article at: http://dx.doi.org/10.21037/atm-20-3042 


\section{Introduction}

Rheumatoid arthritis (RA) is a chronic autoimmune disease that is mainly characterized by progressive arthritis leading to joint damage and dysfunction. Several studies have shown that the secretion of proinflammatory cytokines and bone destruction are closely related to the pathogenesis of RA (1). A large number of studies have found that interleukin- $1 \beta$ (IL-1 $\beta$ ) and tumor necrosis factor- $\alpha$ $(\mathrm{TNF}-\alpha)$ play important roles in the pathogenesis of RA (2). Terkeltaub et al. found that IL-1 $\beta$ and TNF- $\alpha$ can induce cartilage destruction and precipitate bone destruction $(3,4)$. In joints, the balance between bone formation by osteoblasts and bone resorption by osteoclasts is involved in maintaining bone homeostasis (5). The inflammatory microenvironment can activate bone resorption and inhibit the reparative effect of osteoblasts, leading to bone erosion. Danks et al. found that IL- $1 \beta$ and TNF- $\alpha$ can alter bone homeostasis and cause bone destruction (6). Therefore, proinflammatory cytokines and bone homeostasis contribute significantly to the progression of RA.

Metformin (MF) is a common oral hypoglycemic agent mainly used to treat type 2 diabetes. In patients with RA, glucose metabolism affects disease progression (7); it can be reasoned that optimizing glucose metabolism may help to control RA activity. Son et al. found that MF can reduce the inflammation of arthritis by regulating the $\mathrm{T}$ helper 17/ regulatory $\mathrm{T}$ cell (Th17/Treg) balance (8). Furthermore, Chen et al. found that MF can inhibit the proliferation of RA fibroblast-like synoviocytes through the insulinlike growth factor receptor (IGF-IR)/phosphoinositide 3-kinase $(\mathrm{PI} 3 \mathrm{~K}) /$ protein kinase $\mathrm{B}(\mathrm{Akt}) / \mathrm{mammalian}$ target of rapamycin (mTOR) pathway (9). Several studies in vitro have found that MF can inhibit osteoclast activation and induce osteoblast differentiation (10-12). Conversely, MF has also been observed to inhibit osteoblast differentiation (13). Therefore, the mechanism of MF on $\mathrm{RA}$ requires further investigation.

Collagen-induced arthritis (CIA) is the most widely used model to simulate the development of RA. In our study, we investigated the protective effects of MF on systemic inflammation and joints in CIA rats. Detection of serum-related inflammatory factors, histological and immunohistochemical analysis of knee joints, and detection of the expression of related genes in cartilage were performed to elucidate whether MF may be a potential therapeutic agent for RA.
We present the following article in accordance with the ARRIVE reporting checklist (available at http://dx.doi. org/10.21037/atm-20-3042).

\section{Methods}

\section{Animals}

Male Wistar rats (7-8 weeks old) were provided by the Laboratory Animal Center of the Ninth People's Hospital affiliated to Shanghai Jiao Tong University Hospital. They were kept in a specialized environment with independent ventilated cages (IVC) in specific-pathogen-free (SPF) facilities with pathogen-free conditions. The experimental protocol was approved by the Ethics Committee of the Ninth People's Hospital affiliated to Shanghai Jiao Tong University School of Medicine \{No. HKDL[2017]207\}, in compliance with institutional guidelines for the care and use of animals.

\section{Drugs and reagents}

MF was purchased from Bristol-Myers Squibb (Shanghai, China). The $2 \mathrm{mg} / \mathrm{mL}$ bovine type II collagen (CII) and incomplete Freund's adjuvant (IFA) were purchased from Chondrex (Redmond, WA, USA). The primary antibodies of matrix metallopeptidase 9 (MMP-9) and a disintegrin and metalloproteinase with thrombospondin-like motifs 5 (ADAMTS-5) were purchased from Cell Signaling Technology (CST) (Danvers, MA, USA). Rat enzymelinked immunosorbent assay (ELISA) kits (TNF- $\alpha$ and IL-1 $\beta$ ) were purchased from Multi Sciences (Hangzhou, Zhejiang, China). The TRIzol reagent was purchased from Ambion (Shanghai, China). The complementary DNA (cDNA) reverse transcription kit was purchased from Takara Bio (Kusatsu, Shiga, Japan).

\section{Experimental protocols}

We randomly allocated 36 rats into equal groups of three groups: a normal group, a CIA group, and CIA treated with MF group. With the exception of the normal group, the other two groups of rats were immunized. On day 0 , IFA and CII were emulsified at 1:1 and then injected intradermally into the base of the rats' tails. On day 7, the same emulsion was injected in the same way. Beginning on the $14^{\text {th }}$ day, MF $(100 \mathrm{mg} / \mathrm{kg})$ was injected intraperitoneally 
into CIA rats every $3^{\text {rd }}$ day for 3 weeks.

\section{Pharmacodynamic evaluation}

After the first immunization, two independent observers monitored the clinical symptoms of the rats twice a week and measured the volume of the hind paws. At the same time, the severity of arthritis on each paw of the rats was scored from 0 to 4 according to the following system: 0 , normal; 1, mild swelling; 2, moderate swelling at the ankle; 3 , obvious swelling, including ankles, feet, and toes; 4, severe arthritis involving the entire paw.

\section{Determination of TNF- $\alpha$ and $I L-1 \beta$ in peripheral blood}

At the end of the experiment, all rats were anesthetized with an intraperitoneal injection of sodium pentobarbital $(45 \mathrm{mg} / \mathrm{kg})$ and then killed by cervical dislocation. The upper plasma was obtained after peripheral was blood taken and centrifuged at $3,500 \mathrm{rpm}$ for $10 \mathrm{~min}$ at $4{ }^{\circ} \mathrm{C}$. The levels of cytokines (IL-1 $\beta, \mathrm{TNF}-\alpha$ ) in the rat plasma were measured according to the instructions of the ELISA kit.

\section{Microscopic computed tomography (micro-CT) examination}

The hind limbs of the rats were dissected and fixed in 4\% paraformaldehyde (PFA), and then subjected to microCT analysis using the Inveon Micro positron emission tomography (PET)/CT system (Siemens Co., Knoxville, TN, USA). A tomographic scan of the hind limbs was performed with a custom resolution of $10 \mu \mathrm{m}$, a voltage of $70 \mathrm{kV}$, and a current of $114 \mathrm{~mA}$. We reconstructed and analyzed the three-dimensional (3D) structure and morphometry in a double-blind manner. The area between the proximal tibia and the distal femur was selected for analysis of the following parameters: trabecular bone volume fraction (Trab BV/TV), trabecular spacing (Tb.Sp), trabecular number (Tb.N), and trabecular thickness (Tb.Th).

\section{Histopathological analysis of the knee joint}

The hind legs were separated and placed in $4 \%$ PFA for $24 \mathrm{~h}$, and then decalcified with $0.5 \mathrm{M}$ ethylenediamine tetraacetic acid (EDTA) and embedded in paraffin. Paraffin sections with a thickness of $5 \mu \mathrm{m}$ were prepared, dewaxed with xylene, gradiently dehydrated with ethanol, and finally subjected to hematoxylin and eosin (HE), tartrate resistant acid phosphatase (TRAP), alcian blue, and toluidine blue staining. Synovial hyperplasia, inflammatory infiltration, and neovascularization in the knee joint were analyzed by HE staining. The number and area of osteoblasts and osteoclasts in the knee joint were observed by HE and TRAP staining to evaluate the bone destruction of the joint. The cartilage degradation in the knee joint was analyzed by alcian blue and toluidine blue staining.

\section{Immunobistochemistry}

The slices were dewaxed in xylene and hydrated with gradient alcohol. Proteinase $\mathrm{K}$ digestion buffer was added to repair the antigen, and 3\% hydrogen peroxide was added to block endogenous peroxidase. Next, they were blocked with $5 \%$ bovine serum albumin (BSA) and then incubated overnight with the primary antibodies (MMP-9, ADAMTS-5) at $4^{\circ} \mathrm{C}$. The slices were then washed of the primary antibodies and incubated with the secondary antibody at room temperature for $30 \mathrm{~min}$; Dolichos bifloris agglutinin (DBA) was then added for color development. The slides were restained with Mayer's hematoxylin and then installed. The expression level of antibodies in articular cartilage was evaluated by two independent observers.

\section{Real-time polymerase-chain reaction (RT-PCR)}

Total RNA was extracted from the rat femoral head using the TRIzol reagent, and the concentration and purity were measured. The cDNA reverse transcription kit was used to reverse-transcribe into cDNA according to standard procedures. The RT-PCR primers were synthesized by Shanghai Sangon Biological Engineering Technology \& Services Co., Ltd. The primers were as follows: caspase-3 (Casp3) (F): 5'-GGAGCTTGGAACGCGAAGAA-3' and (R): 5'-ACACAAGCCCATTTCAGGGT-3'; Bcl-2 associated X protein (Bax) (F): 5'-TTGCTACAGGGTTTCATCCAGG-3' and (R): 5'-CACTCGCTCAGCTTCTTGGT-3'; protein 53 (p53) (F): 5'-CCCCTGAAGACTGGATAACTGT-3' and (R): 5'-CACTTGGAGGGCTTCCTCTG-3'. Gene expression levels of Casp3, Bax, and p53 were measured via RT-PCR (ABI 7500, Applied Biosystems Inc., USA), according to the manufacturer's protocols.

\section{Statistical analysis}

The results are expressed as mean \pm standard deviation (SD). 
A

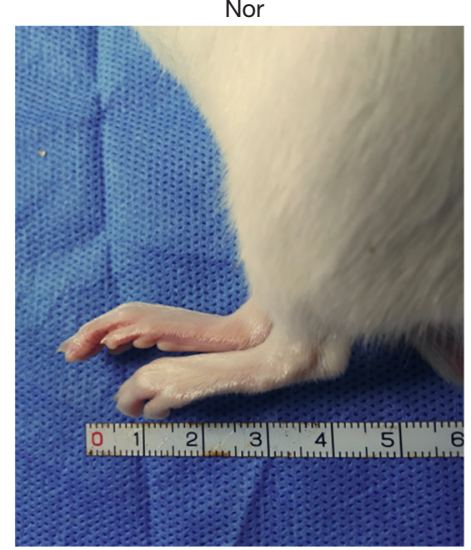

$\mathrm{CIA}$

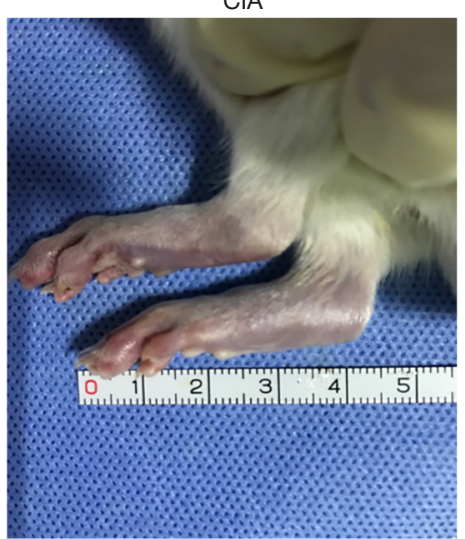

MF

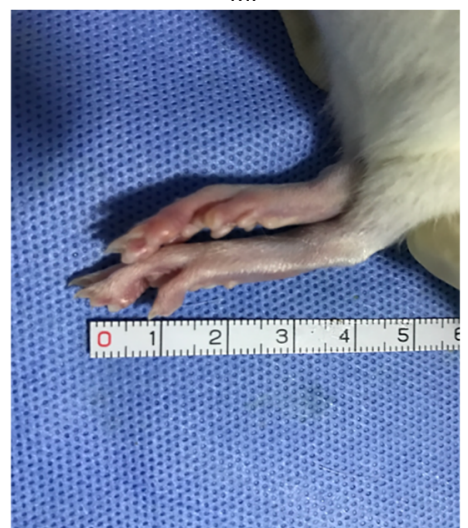

B
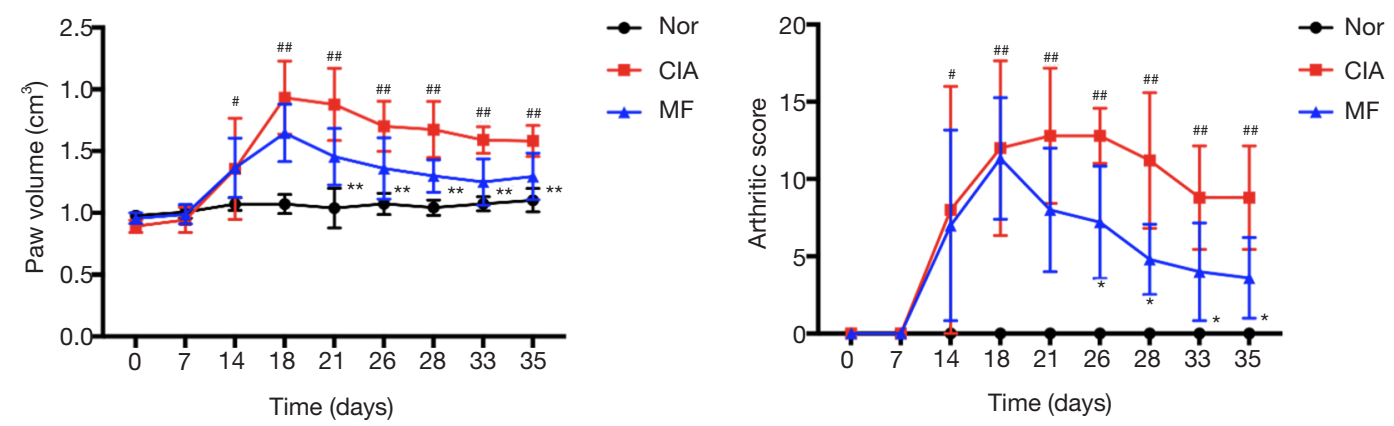

Figure 1 Protective effect of MF on CIA rats. (A) Representative images of paw swelling in the normal, CIA, and MF group on the day after the end of the administration; (B) the paw swelling and arthritic scores of the mice in the normal, CIA, and MF group. The CIA group showed significant paw swelling from day 14. After MF treatment, the swelling of the ankle joint was significantly relieved from day 21 onwards. Arthritic score revealed that the arthritis symptoms of the CIA group began on day 14 and peaked around day 21. After MF treatment, arthritis symptoms were significantly relieved from day 26 . All data are expressed as mean $\pm \mathrm{SD}$. ${ }^{\#}, \mathrm{P}<0.05,{ }^{\#}, \mathrm{P}<0.01$ the CIA group vs. the normal group; ${ }^{*}, \mathrm{P}<0.05,{ }^{* *}, \mathrm{P}<0.01$ the MF group $v s$. the CIA group. MF, metformin; CIA, collagen-induced arthritis; SD, standard deviation.

The statistical significance between various groups was analyzed using one-way analysis of variance (ANOVA) in SPSS 22.0 software. Differences between groups of $\mathrm{P}<0.05$ were considered statistically significant.

\section{Results}

\section{MF inbibited the progression of arthritis and systemic inflammation in CIA rats}

No rat death was observed during the course of the experiment. Beginning the $10^{\text {th }}$ day, the paws of the immunized rats presented as obviously red and swollen and reached a peak on the $18^{\text {th }}$ day. Beginning the $14^{\text {th }}$ day, MF was injected intraperitoneally into the CIA rats every 3 days for 3 weeks. Following treatment with MF, arthritis symptoms were significantly reduced (Figure $1 A$ ). The CIA group showed significant paw swelling from day 14 . After administration of MF, the ankle joint swelling was significantly reduced from day 21 (Figure $1 B$ ). As revealed by the arthritic score, the arthritis symptoms of the CIA group were apparent on the $14^{\text {th }}$ day and reached a peak at around day 21 . After MF treatment, arthritic symptoms were significantly relieved from day 26 (Figure $1 B$ ). After completion of administration, the levels of pro-inflammatory cytokines (IL-1 $\beta, T N F-\alpha$ ) in the peripheral plasma were measured by ELISA test. The levels of IL-1 $\beta$ and TNF- $\alpha$ in the peripheral blood of the CIA group were significantly increased, but they were significantly reduced in the MF group (Figure $2 A$ ). Therefore, these results demonstrate that MF has a significant inhibitory effect on arthritis symptoms and 

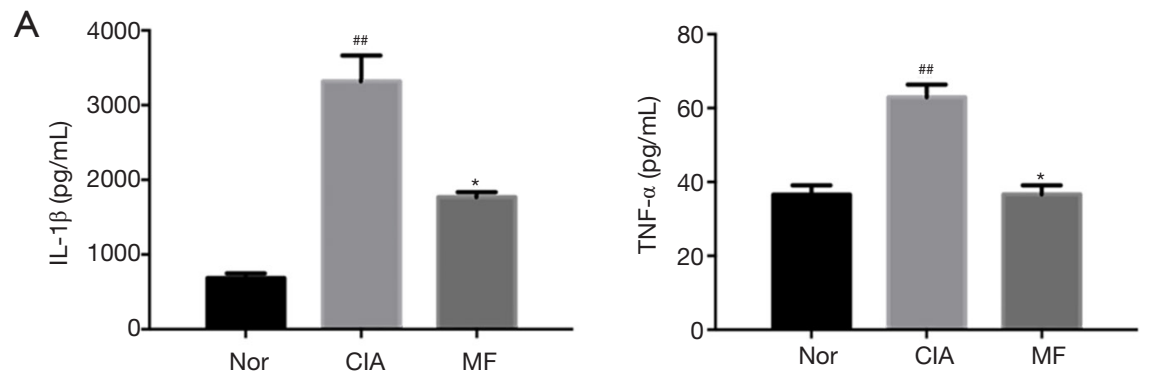

B
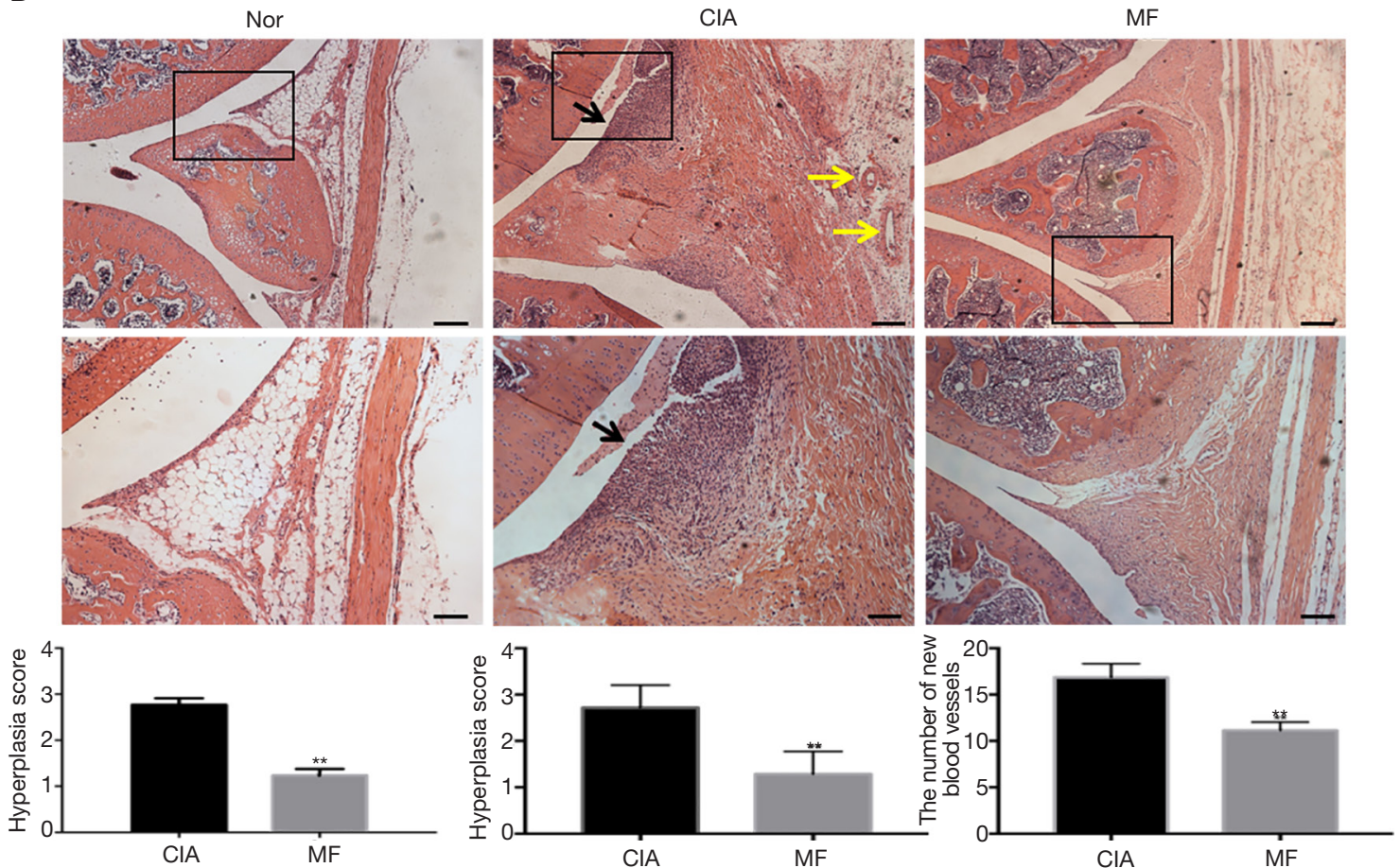

Figure 2 Effects of MF on arthritis and systemic inflammation in CIA rats. Representative knee joint sections stained with hematoxylin and eosin. The black boxes represent areas of magnification in the corresponding figure below. (A) Cytokine levels in rat peripheral plasma. The levels of proinflammatory cytokines (IL-1 $\beta, \mathrm{TNF}-\alpha$ ) in peripheral blood in the CIA group were significantly increased, but they were significantly reduced in the MF group; (B) effect of MF on the synovial membrane of the knee joints of CIA rats. Compared with the joints of normal rats, CIA rats showed obvious symptoms of RA, such as synovial hyperplasia, inflammatory cell infiltration (black arrow), and neovascularization (yellow arrow). After MF treatment, synovial hyperplasia, inflammatory infiltration, and neovascularization were significantly inhibited in CIA rats. Scale bar, $200 \mu \mathrm{m}$ (upper panel) and $100 \mu \mathrm{m}$ (lower panel). All data are expressed as mean $\pm \mathrm{SD}$. ${ }^{\#}, \mathrm{P}<0.01$ the CIA group vs. the normal group; ${ }^{*}, \mathrm{P}<0.05,{ }^{* *}, \mathrm{P}<0.01$ the $\mathrm{MF}$ group vs. the CIA group. MF, metformin; CIA, collagen-induced arthritis; IL-1 $\beta$, interleukin-1 $\beta$; TNF- $\alpha$, tumor necrosis factor- $\alpha$; RA, rheumatoid arthritis; SD, standard deviation.

systemic inflammation in CIA rats.

\section{MF inhibited synovial byperplasia, inflammatory infiltration, and neovascularization in CIA rats}

Compared with the joints of the normal rats, CIA rats showed obvious symptoms of RA, such as synovial hyperplasia, inflammatory cell infiltration, and neovascularization. Histological analysis found that MF improved arthritis symptoms in CIA rats. The histological score of the synovium showed that MF significantly inhibited synovial hyperplasia, inflammatory infiltration, 

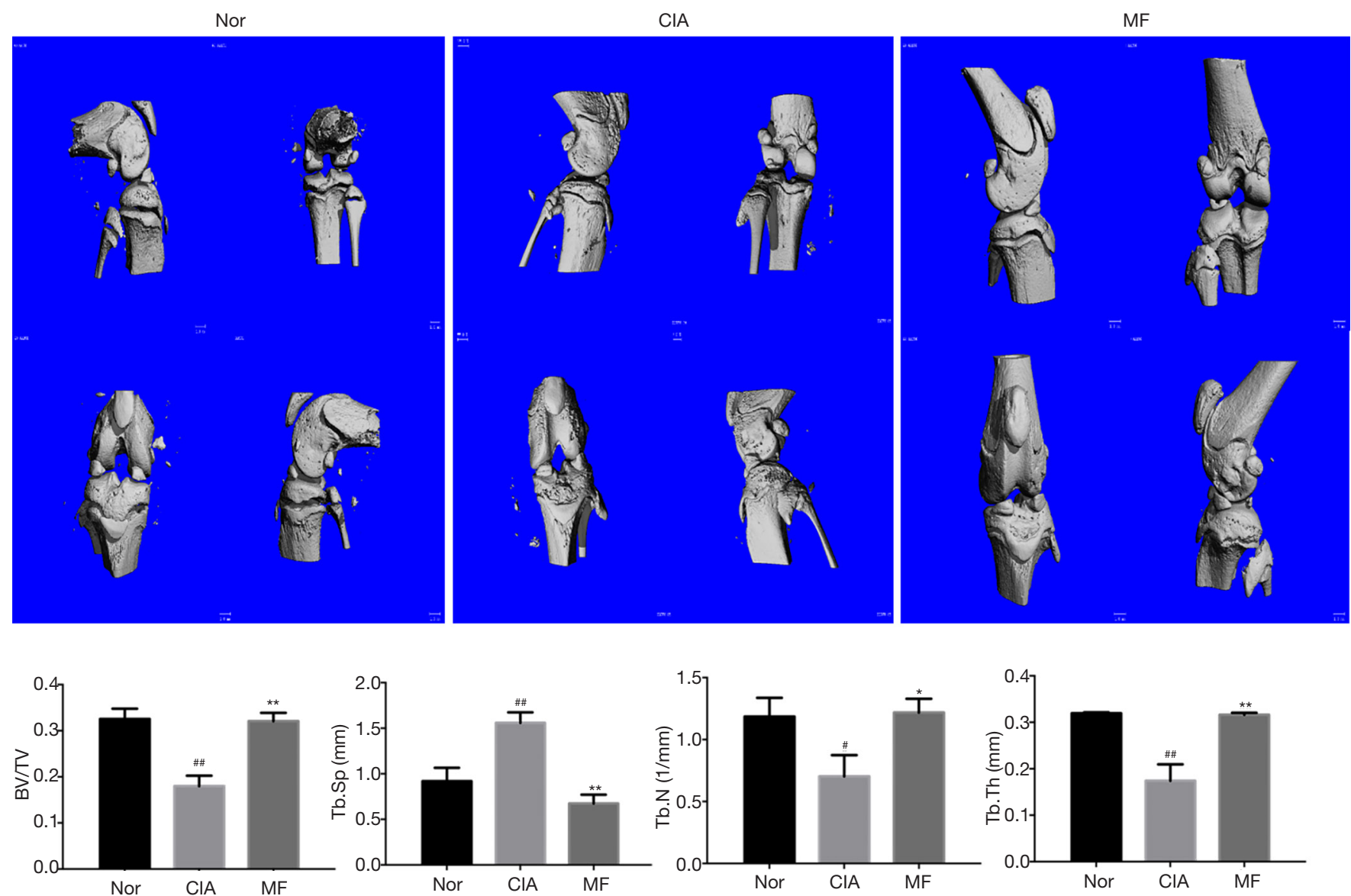

Figure 3 Effects of MF on joint bone erosion in CIA rats. Reconstructed 3D micro-CT images of knee joints of the normal, CIA, and MF group rats to show joint bone erosion. The values of BV/TV, Tb.N, and Tb.Th of the CIA group were downregulated, and the values of Tb.Sp were upregulated. After 3 weeks of MF treatment, the values of BV/TV, Tb.N, and Tb.Th were significantly upregulated, and the values of Tb.Sp were significantly reduced. All data are expressed as mean $\pm \mathrm{SD}$. ${ }^{\#}, \mathrm{P}<0.05,{ }^{\#}, \mathrm{P}<0.01$ the CIA group vs. the normal group; *, $\mathrm{P}<0.05$, **, $\mathrm{P}<0.01$ the MF group vs. the CIA group. MF, metformin; CIA, collagen-induced arthritis; 3D, three-dimensional; microCT, microscopic computed tomography; BV/TV, bone volume fraction; Tb.N, trabecular number; Tb.Th, trabecular thickness; Tb.Sp, trabecular spacing; SD, standard deviation.

and neovascularization in CIA rats (Figure 2B).

\section{MF improved the joint bone destruction in CIA rats}

Compared with the joints of normal rats, there was significant bone destruction in the knee joints of CIA rats. After treatment with MF, the bone destruction phenomenon was significantly improved. From the parameter analysis of rat knee micro-CT, it could be seen that the values of BV/TV, Tb.N and Tb.Th of the CIA group were downregulated, and the values of Tb.Sp were upregulated. After 3 weeks of MF treatment, the values of BV/TV, Tb.N, and $\mathrm{Tb}$.Th were significantly upregulated, and the values of Tb.Sp were significantly reduced (Figure 3). Osteoblasts play a very important role in regulating bone metabolism. Compared to normal rats, there was a significant decline in osteoblasts in the region of knee joint in CIA rats. There was no significant improvement observed in osteoblast loss in the regions of subchondral bone and trabecular bone after the administration of MF (Figure 4). Osteoclast formation is a major problem in RA. Compared to normal rats, there was a significant increase in osteoclasts in the knee joint region of CIA rats. After treatment with MF, the proliferation of osteoclasts in cartilage and trabecular 
A

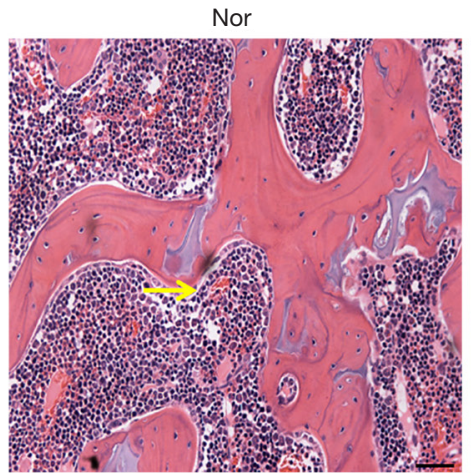

B
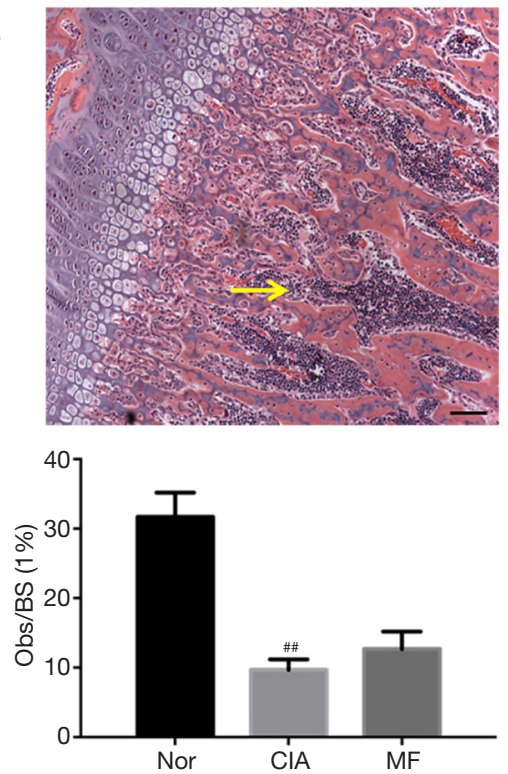

CIA
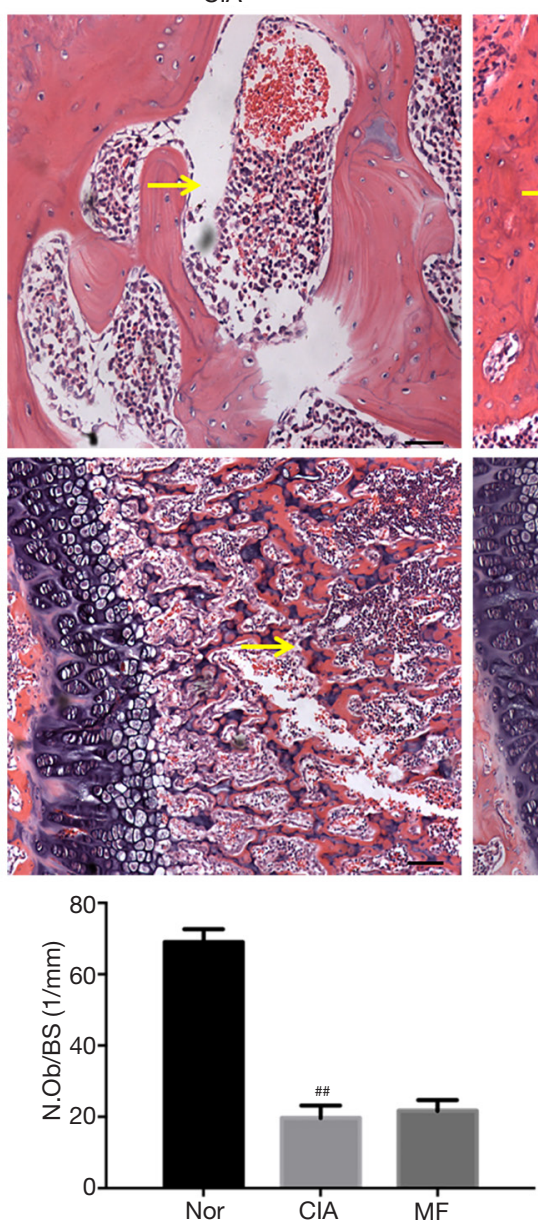
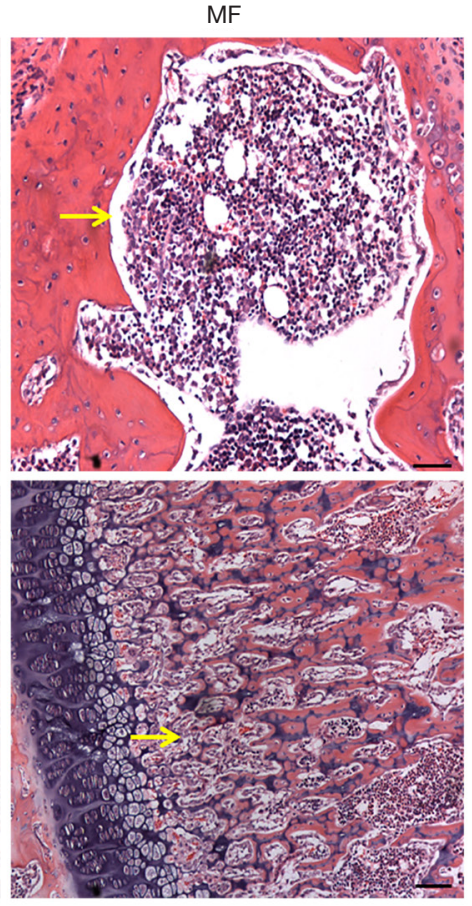

Figure 4 Effect of MF on osteoblasts in joints of CIA rats. Representative knee joint sections stained with hematoxylin and eosin. Compared to normal rats, there was a significant loss of osteoblasts (yellow arrow) in the region of the knee joint in CIA rats. There was no significant improvement in osteoblast loss in the regions of subchondral bone (A) and trabecular bone (B) after administration of MF. Scale bar, $50 \mu \mathrm{m}$ (upper panel) and $100 \mu \mathrm{m}$ (lower panel). All data are expressed as mean $\pm \mathrm{SD} .{ }^{\# \#}, \mathrm{P}<0.01$ the CIA group vs. the normal group. MF, metformin; CIA, collagen-induced arthritis; SD, standard deviation.

bone was significantly alleviated (Figure 5). Therefore, MF can play a bone protective role by inhibiting osteoclast formation.

\section{MF inbibited cartilage destruction in CIA rats}

Damage of the articular cartilage is another important factor that contributes to bone destruction. Compared to normal rats, there was significant degradation of the cartilage layer of the knee joint in CIA rats. After treatment with $\mathrm{MF}$, the cartilage damage was significantly alleviated (Figure 6). The roles of enzymes MMP-9 and ADAMTS-5 are very important in the degradation of articular cartilage.
Compared with the normal group, the expression of MMP-9 and ADAMTS-5 in cartilage of CIA rats was significantly increased, but was decreased following treatment with MF (Figure $7 A, B$ ). Casp3, Bax and p53, are important in regulating apoptosis of chondrocytes. Accordingly, we analyzed their expression in chondrocytes by RTPCR. Compared to normal rats, the mRNA expression of Casp3, Bax, and p53 in the chondrocytes of CIA rats was significantly higher (Figure 7C). Nevertheless, the mRNA expression of Casp3, Bax, and p53 was significantly decreased after MF treatment. Therefore, MF was seen to have a protective effect against apoptosis of chondrocytes in CIA rats. 
A

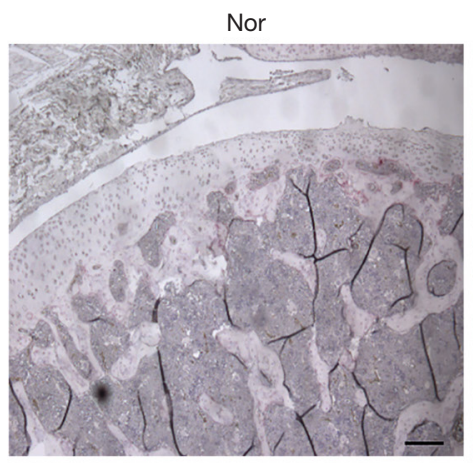

B
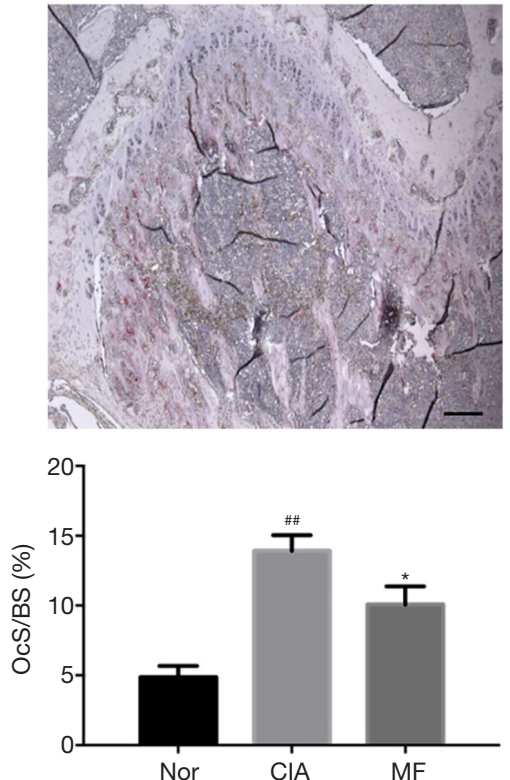

CIA
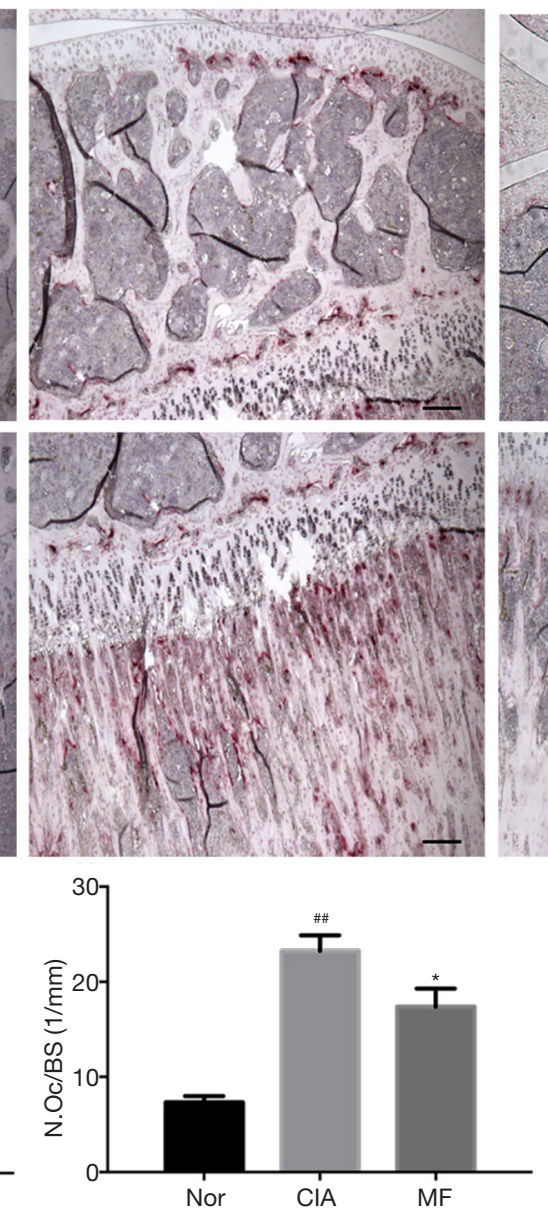
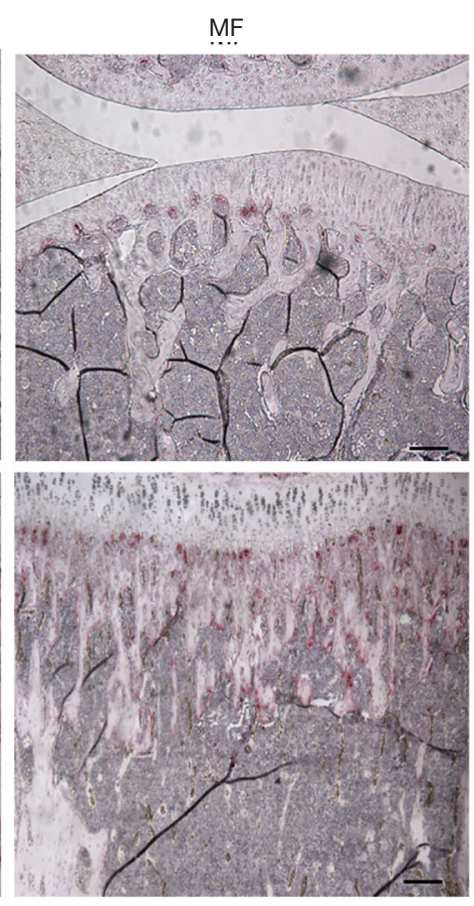

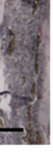

. 

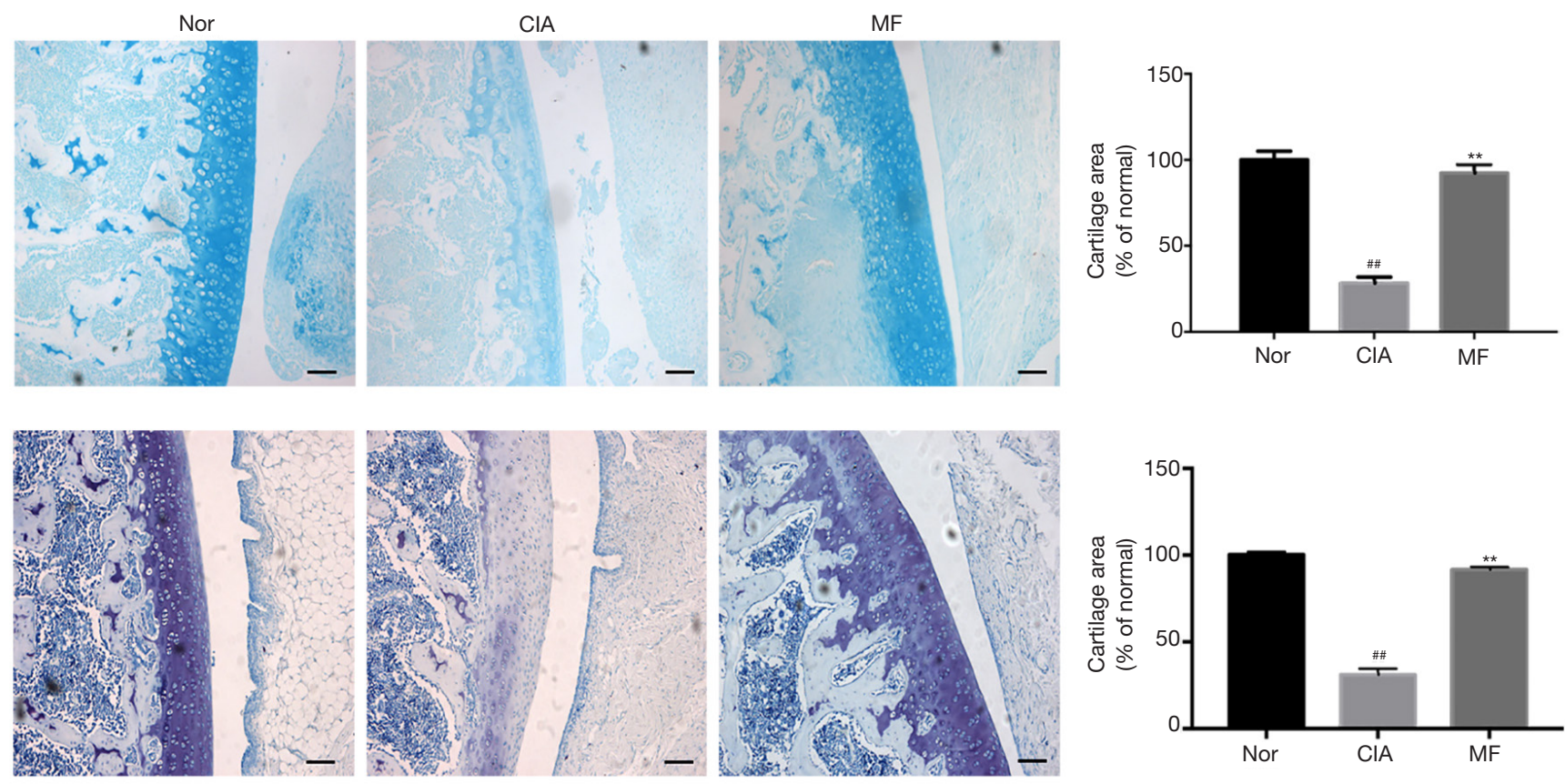

Figure 6 Effect of MF on cartilage in joints of CIA rats. Representative knee joint sections stained with Alcian blue (upper panel) and Toluidine blue (lower panel). Compared to normal rats, the cartilage layer of the knee joint in CIA rats was significantly degraded. After treatment with MF, the cartilage damage was significantly alleviated. Scale bar, $50 \mu \mathrm{m}$. All data are expressed as mean $\pm \mathrm{SD}$. ${ }^{\#}, \mathrm{P}<0.01$ the CIA group vs. the normal group; ${ }^{* *}, \mathrm{P}<0.01$ the MF group vs. the CIA group. MF, metformin; CIA, collagen-induced arthritis; SD, standard deviation.

of the knee joint revealed that synovial hyperplasia was suppressed, inflammatory infiltration was reduced, and neovascularization was controlled after MF treatment. In summary, our results indicated that MF can significantly inhibit systemic inflammation and synovitis in CIA rats.

In addition to inflammation, CIA rats also exhibit severe bone erosion. In order to determine whether MF can protect bones against the erosion caused by arthritis, we analyzed the knee joint microstructure by micro-CT and histomorphometry. Studies have reported that the number, volume, and thickness of trabeculae of CIA rats are significantly reduced, and the distance between trabeculae is increased. In our study, after MF treatment was applied, the CIA-related bone destruction was significantly suppressed. The severity of bone destruction is closely related to the balance between osteoblasts and osteoclasts (19). Staining of the knee joint with HE revealed that severe osteoblast loss had occurred in the subchondral bone and trabecular bone area of CIA rats, and it was not significantly improved after MF treatment. Staining of the knee joint with TRAP revealed that osteoclasts in the subchondral bone and trabecular bone region of CIA rats were significantly increased, and were significantly inhibited after MF treatment. Therefore, our results indicated that MF can exert bone protection by inhibiting osteoclast formation.

Damage to the articular cartilage is also an important cause of bone destruction (20,21). Cartilage is mainly composed of chondrocytes and extracellular matrix (ECM). The composition of ECM is mainly interstitial fluid, collagen, and proteoglycans. Under physiological conditions, the balance of chondrocytes and ECM maintains the homeostasis of cartilage (22). However, in the case of RA, this steady state is interrupted. Alcian blue and toluidine blue staining of the knee joints revealed that the cartilage layer of CIA rats had become significantly degraded, and was improved after treatment with MF. In cartilage damage, ADAMTS and matrix metalloproteinases (MMPs) all play important roles in the degradation of ECM (23). The most important enzyme in MMPs is MMP-9; it is highly expressed in arthritis, can specifically degrade collagen, and its reduction can ameliorate the progress of arthritis $(24,25)$. ADAMTS-5 is considered the most important enzyme 
A
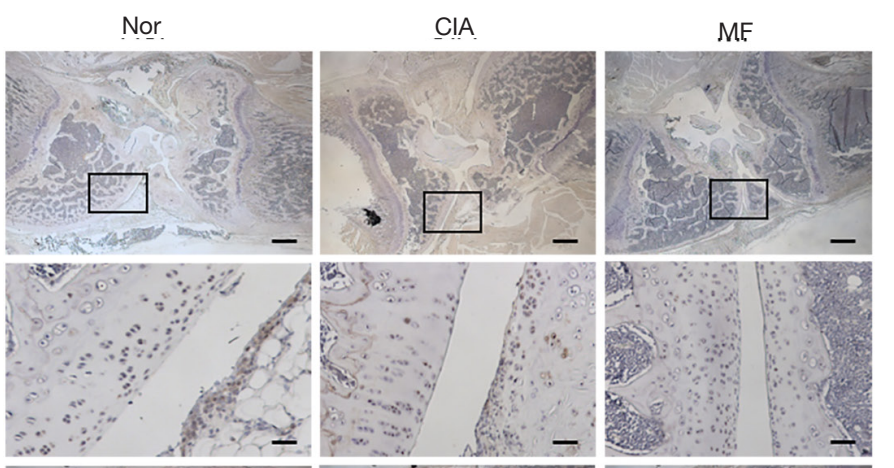

B
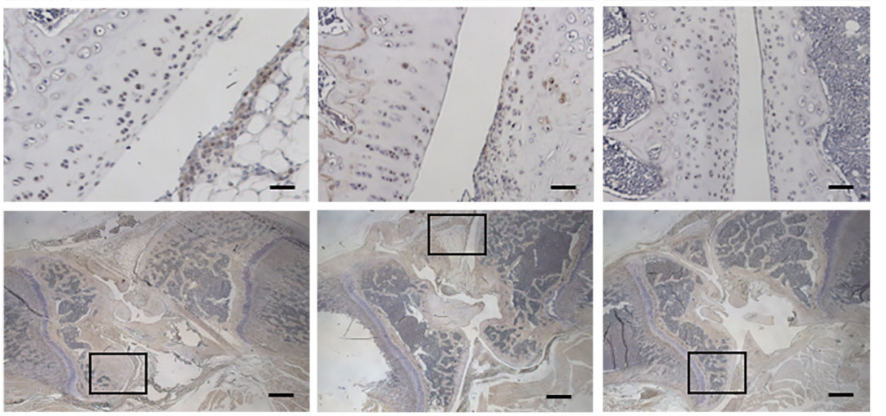

C
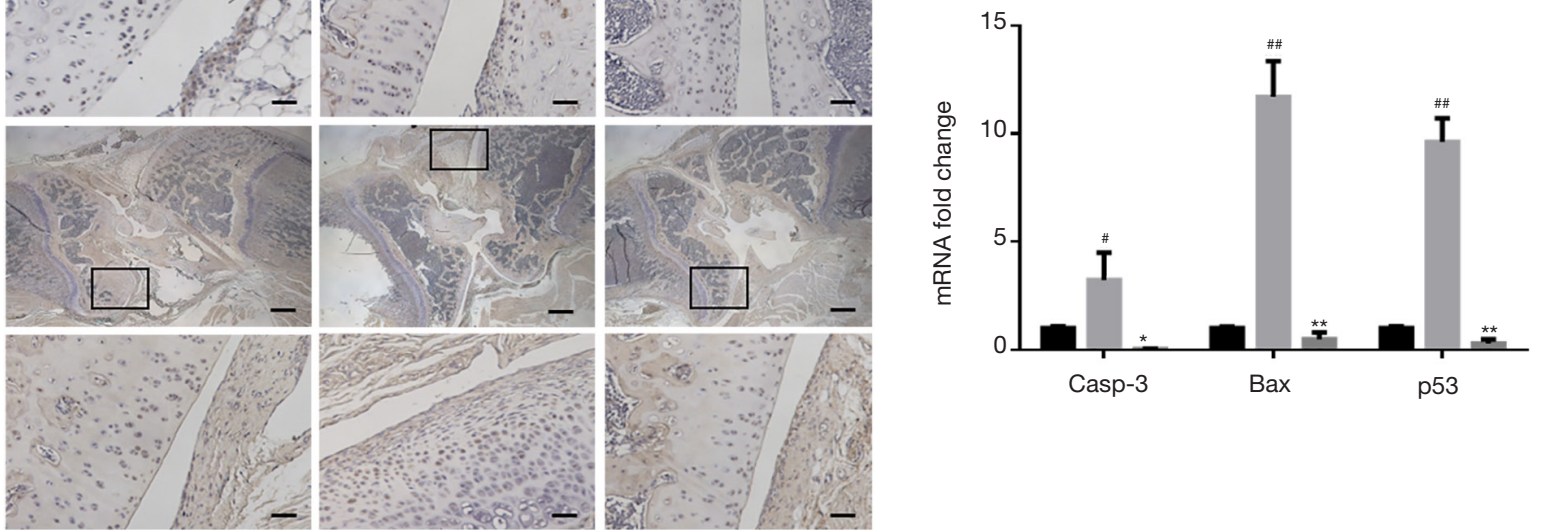

Figure 7 Effects of MF on ADAMTS-5 (A), MMP-9 (B) in cartilage of the knee joints in CIA rats. Representative knee joint sections were processed by immunohistochemistry. The black boxes represent areas of magnification in the corresponding figure below. Compared with the normal group, the expression of MMP-9 and ADAMTS-5 in cartilage of CIA rats was significantly increased, but decreased after MF treatment; (C) effects of MF on the expression of Casp3, Bax and p53 in chondrocytes in CIA rats. The mRNA expression of Casp3, Bax and $\mathrm{p} 53$ in chondrocytes in CIA rats was significantly higher than that in normal rats. Nevertheless, the mRNA expression of Casp3, Bax and $\mathrm{p} 53$ was significantly decreased after MF treatment. Scale bar, $200 \mu \mathrm{m}$ (upper panel) and $50 \mu \mathrm{m}$ (lower panel). All data are expressed as mean $\pm \mathrm{SD}$. ${ }^{\prime}, \mathrm{P}<0.05,{ }^{* \#}, \mathrm{P}<0.01$ the CIA group $v s$. the normal group; ${ }^{*}, \mathrm{P}<0.05,{ }^{* *}, \mathrm{P}<0.01$ the MF group $v s$. the CIA group. MF, metformin; ADAMTS-5, a disintegrin and metalloproteinase with thrombospondin-like motifs 5; MMP-9, matrix metallopeptidase 9; CIA, collageninduced arthritis; Casp3, caspase-3; Bax, Bcl-2 associated X protein; p53, protein 53; SD, standard deviation.

for degrading proteoglycans in ADAMTS (26). Chen et al. found that ADAMTS-5 inhibitors can significantly inhibit cartilage degeneration in arthritis (27). In our study, the expression of MMP-9 and ADAMTS-5 in the cartilage layer of CIA rats was increased, and was then significantly inhibited after MF treatment. In addition to the degradation of ECM, chondrocyte apoptosis causes cartilage degeneration (28). Bax is a well-known proapoptotic protein of the B-cell lymphoma-2 (Bcl-2) family, and is thought to play an important role in chondrocyte apoptosis (28). Belonging to the cysteine protease family, Casp3 is considered to be an important mediator of nitric oxide (NO)-induced chondrocyte apoptosis (29). The tumor suppressor gene p53 plays a vital role in regulating bone and chondrocyte apoptosis (30). In our study, the expression of related pro-apoptotic genes (Bax, Casp3, and p53) in chondrocytes in CIA rats was significantly increased, and the viability of chondrocytes was significantly increased after MF treatment. In summary, our results indicate that MF can significantly inhibit cartilage degeneration induced by CIA.

\section{Conclusions}

Overall, this study clearly showed that MF can inhibit systemic inflammation and synovitis, and protect bone by inhibiting ECM degradation, osteoclast formation, and chondrocyte apoptosis. Our results suggest that MF may be a potential drug for the treatment of RA.

\section{Acknowledgments}

Funding: This work was supported by the National Natural Science Foundation of China [Grants 81874011, 81572104 , and 81301531 (to TYW)]. This work was also 
partially supported by the Shanghai Municipal Science and Technology Commission [Innovation Grant 18140903502 (to TYW)].

\section{Footnote}

Reporting Checklist: The authors have completed the ARRIVE reporting checklist. Available at http://dx.doi. org/10.21037/atm-20-3042

Data Sharing Statement: Available at http://dx.doi. org/10.21037/atm-20-3042

Conflicts of Interest: All authors have completed the ICMJE uniform disclosure form (available at http://dx.doi. org/10.21037/atm-20-3042). The authors have no conflicts of interest to declare.

Ethical Statement: The authors are accountable for all aspects of the work in ensuring that questions related to the accuracy or integrity of any part of the work are appropriately investigated and resolved. The experimental protocol was approved by the Ethics Committee of the Ninth People's Hospital affiliated to Shanghai Jiao Tong University School of Medicine \{No. HKDL[2017]207\}, in compliance with institutional guidelines for the care and use of animals.

Open Access Statement: This is an Open Access article distributed in accordance with the Creative Commons Attribution-NonCommercial-NoDerivs 4.0 International License (CC BY-NC-ND 4.0), which permits the noncommercial replication and distribution of the article with the strict proviso that no changes or edits are made and the original work is properly cited (including links to both the formal publication through the relevant DOI and the license). See: https://creativecommons.org/licenses/by-nc-nd/4.0/.

\section{References}

1. Hammaker D, Firestein GS. Epigenetics of inflammatory arthritis. Curr Opin Rheumatol 2018;30:188-96.

2. Huang CC, Chiou CH, Liu SC, et al. Melatonin attenuates TNF- $\alpha$ and IL- $1 \beta$ expression in synovial fibroblasts and diminishes cartilage degradation: Implications for the treatment of rheumatoid arthritis. J Pineal Res 2019;66:e12560.

3. Terkeltaub R, Yang B, Lotz M, et al. Chondrocyte
AMP-activated protein kinase activity suppresses matrix degradation responses to proinflammatory cytokines interleukin- $1 \beta$ and tumor necrosis factor $\alpha$. Arthritis Rheum 2011;63:1928-37.

4. Petursson F, Husa M, June R, et al. Linked decreases in liver kinase $\mathrm{B} 1$ and $\mathrm{AMP}$-activated protein kinase activity modulate matrix catabolic responses to biomechanical injury in chondrocytes. Arthritis Res Ther 2013;15:R77.

5. Tanaka Y, Okada Y, Nakamura T. Inter- and intracellular signaling in secondary osteoporosis. J Bone Miner Metab 2003;21:61-6.

6. Danks L, Komatsu N, Guerrini MM, et al. RANKL expressed on synovial fibroblasts is primarily responsible for bone erosions during joint inflammation. Ann Rheum Dis 2016;75:1187-95.

7. Dessein PH, Joffe BI. Insulin resistance and impaired beta cell function in rheumatoid arthritis. Arthritis Rheum 2006;54:2765-75.

8. Son HJ, Lee J, Lee SY, et al. Metformin attenuates experimental autoimmune arthritis through reciprocal regulation of Th17/Treg balance and osteoclastogenesis. Mediators Inflamm 2014;2014:973986.

9. Chen K, Lin ZW, He SM, et al. Metformin inhibits the proliferation of rheumatoid arthritis fibroblastlike synoviocytes through IGF-IR/PI3K/AKT/m-TOR pathway. Biomed Pharmacother 2019;115:108875.

10. Shah M, Kola B, Bataveljic A, et al. AMP-activated protein kinase (AMPK) activation regulates in vitro bone formation and bone mass. Bone 2010;47:309-19.

11. Lee YS, Kim YS, Lee SY, et al. AMP kinase acts as a negative regulator of RANKL in the differentiation of osteoclasts. Bone 2010;47:926-37.

12. Wang P, Ma T, Guo D, et al. Metformin induces osteoblastic differentiation of human induced pluripotent stem cell-derived mesenchymal stem cells. J Tissue Eng Regen Med 2018;12:437-46.

13. Zhen D, Chen Y, Tang X, et al. Metformin reverses the deleterious effects of high glucose on osteoblast function. J Diabetes Complications 2010;24:334-44.

14. Karouzakis E, Gay RE, Gay S, et al. Epigenetic control in rheumatoid arthritis synovial fibroblasts. Nat Rev Rheumatol 2009;5:266-72.

15. Leblond A, Allanore Y, Avouac J. Targeting synovial neoangiogenesis in rheumatoid arthritis. Autoimmun Rev 2017;16:594-601.

16. García-Vicuña R, Gómez-Gaviro MV, Domínguez-Luis $\mathrm{MJ}$, et al. CC and CXC chemokine receptors mediate migration, proliferation, and matrix metalloproteinase 
production by fibroblast-like synoviocytes from rheumatoid arthritis patients. Arthritis Rheum 2004;50:3866-77.

17. Li J, Zhang B, Liu WX, et al. Metformin limits osteoarthritis development and progression through activation of AMPK signaling. Ann Rheum Dis 2020;79:635-45.

18. Wu J, Zhao FT, Fan KJ, et al. Dihydromyricetin inhibits inflammation of fibroblast-like synoviocytes through regulation of nuclear factor-kb signaling in rats with collagen-induced arthritis. J Pharmacol Exp Ther 2019;368:218-28.

19. Rajaei E, Haybar H, Mowla K, et al. Metformin one in a million efficient medicines for rheumatoid arthritis complications: inflammation, osteoblastogenesis, cardiovascular disease, malignancies. Curr Rheumatol Rev 2019;15:116-22.

20. Smolen JS, Aletaha D, McInnes IB. Rheumatoid arthritis. Lancet 2016;388:2023-38.

21. Pap T, Korb-Pap A. Cartilage damage in osteoarthritis and rheumatoid arthritis--two unequal siblings. Nat Rev Rheumatol 2015;11:606-15.

22. Yasuda T. Cartilage destruction by matrix degradation products. Mod Rheumatol 2006;16:197-205.

23. Noss EH, Brenner MB. The role and therapeutic implications of fibroblast-like synoviocytes in inflammation and cartilage erosion in rheumatoid arthritis. Immunol Rev 2008;223:252-70.

Cite this article as: Fan KJ, Wu J, Wang QS, Xu BX, Zhao FT, Wang TY. Metformin inhibits inflammation and bone destruction in collagen-induced arthritis in rats. Ann Transl Med 2020;8(23):1565. doi: 10.21037/atm-20-3042
24. Tchetverikov I, Ronday HK, Van EIB, et al. MMP profile in paired serum and synovial fluid samples of patients with rheumatoid arthritis. Ann Rheum Dis 2004;63:881-3.

25. Burrage PS, Mix KS, Brinckerhoff CE. Matrix metalloproteinases: Role in arthritis. Front Biosci 2006;11:529-43.

26. Stanton H, Rogerson FM, East CJ, et al. ADAMTS5 is the major aggrecanase in mouse cartilage in vivo and in vitro. Nature 2005;434:648-52.

27. Chen P, Zhu S, Wang Y, et al. The amelioration of cartilage degeneration by ADAMTS-5 inhibitor delivered in a hyaluronic acid hydrogel. Biomaterials 2014;35:2827-36.

28. Miao G, Zang X, Hou H, et al. Bax targeted by miR-29a regulates chondrocyte apoptosis in osteoarthritis. Biomed Res Int 2019;2019:1434538.

29. Ueng SW, Yuan LJ, Lin SS, et al. Hyperbaric oxygen treatment prevents nitric oxide-induced apoptosis in articular cartilage injury via enhancement of the expression of heat shock protein 70. J Orthop Res 2013;31:376-84.

30. Liu B, Lei M, Hu T, et al. Inhibitory effects of SRT1720 on the apoptosis of rabbit chondrocytes by activating

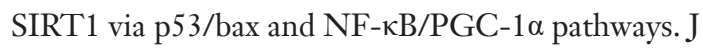
Huazhong Univ Sci Technolog Med Sci 2016;36:350-5.

(English Language Editors: J. Jones and J. Gray) 